\title{
Distribution of Abnormal Laboratory Tests in Patients with Dehydration Due to Gastroenteritis: A Medical Audit Study
}

\author{
Parsa Yousefichaijan, ${ }^{1}$ Fatemeh Dorreh, ${ }^{2}$ Elahe Ziaei, ${ }^{3}$ and Abdolghader Pakniyat ${ }^{4,}$ \\ ${ }^{1}$ Associate Professor of Pediatric Nephrology, School of Medicine, Arak University of Medical Sciences, Arak, Iran \\ ${ }^{2}$ Assistant Professor of Pediatrics, School of Medicine, Arak University of Medical Sciences, Arak, Iran \\ ${ }^{3} \mathrm{MD}$, Arak University of Medical Sciences, Arak, Iran \\ ${ }^{4}$ MD, Student Research Committee, Emergency Medicine Department, Arak University of Medical Sciences, Arak, Iran \\ "Corresponding author: Abdolghader Pakniyat, Student Research Committee, Emergency Medicine Department, Arak University of Medical Sciences, Arak, Iran, E-mail: \\ abdolghader.pakniyat@gmail.com
}

Received 2016 April 11; Revised 2016 September 23; Accepted 2016 November 28.

\begin{abstract}
Background: Gastroenteritis is one of the major causes of morbidity and mortality among children, and it accounts for $18 \%$ of childhood death. Conducting laboratory tests is challenging for these patients, and the validity of the laboratory studies in treatment and prognosis is in question. Objectives: This study aimed to investigate the necessity of performing laboratory tests, and intended to reduce hospitalization costs.

Methods: Patients with moderate to severe dehydration due to gastroenteritis, who referred to the emergency ward of Amir Kabir hospital from April to August 2008, were enrolled in this cross- sectional descriptive study. The routine lab tests, including complete blood cell, urine analysis, stool exam and electrolytes were performed, and statistical analysis was done by SPSS software.

Results: Of the 610 patients, $0.3 \%$ had severe, $99.7 \%$ had moderate dehydration, and $42.78 \%$ were female. The lab results of the patients revealed the following information: Blood sugar: 97.3\%; sodium: 95.5\%; potassium: 93.5\%; blood creatinine: $95.4 \%$; blood urea nitro: $87.9 \%$; urine analysis: $90.7 \%$; urine culture: 96.8\%; stool exam: 83.3\%; white blood cell count: $87.7 \%$. However, hemoglobin was found to be $77.4 \%$, which was normal.

Conclusions: It is reasonable to evaluate blood urea nitrogen, potassium, and hemoglobin of children with gastroenteritis. Moreover, conducting white blood cell, stool exam, and urine analysis is recommended for those with febrile gastroenteritis, especially in girls younger than two years of age. Overall, conducting all the mentioned tests for these patients is not necessary as a routine, and decision should be made based on the clinical condition of the patients and their doctors' opinion.
\end{abstract}

Keywords: Gastroenteritis, Dehydration, Pediatric

\section{Background}

Gastroenteritis is one of the major causes of morbidity and mortality among children, and it is responsible for $18 \%$ of deaths in children. The prevalence of diarrhea for each child is estimated to be 3.2 times per year (1). In the U.S., 200 thousands hospitalization and 300 deaths related to diarrhea are reported annually (1). Approximately, 80\% of its mortality occurs in children less than two years old, and dehydration is the major cause of death. On the other hand, inappetence is a side effect of diarrhea, resulting in malnutrition and growth impairment $(1,2)$.

Gastroenteritis is caused by varying pathogens such as bacteria, viruses and parasites; its presentation is different depending on the organism and host's defense mechanism, and it may present with fever or one of the following symptoms: Watery diarrhea, dysentery, chronic diarrhea, vomiting, abdominal cramps and extraintestinal symptoms $(3,4)$. The diagnosis can be established by epidemiological evidence, clinical symptoms, or physical examination $(5,6)$. The diarrhea attacks could be diminished spontaneously and performing extra laboratory assessments is not necessary in outpatient settings. However, it is controversial to perform Initial laboratory tests such as complete blood count, electrolytes, BUN and $\mathrm{Cr}$ and analysis of urine and stool on hospitalized patients $(1,7)$.

Gastroenteritis is one of the major causes of morbidity and mortality among children, and it accounts for $18 \%$ of childhood death. Conducting laboratory tests is challenging for these patients, and the validity of the laboratory studies in treatment and prognosis is in question.

\section{Objectives}

This study aimed to investigate the necessity of performing laboratory tests being routinely requested for patients with gastroenteritis and compare the results with other studies (Medical Audit) to enhance patients' treatment and reduce the expenditure and hospitalization costs.

\section{Methods}

In this cross-sectional prospective and descriptive study, those children with moderate to severe dehydration, who referred to Amir Kabir hospital from April to August 2008 due to gastroenteritis, were enrolled. Initially, history and physical examination were done accurately and 
then routine laboratory tests, including complete blood red cell count, glucose, sodium level, potassium level, blood urea nitrogen, creatinine, urine culture and stool examination were conducted. All children with moderate to severe dehydration due to gastroenteritis were included. However, those patients who were discharged from the hospital with outpatient therapy, including ORS, or antibiotics or those whose laboratory tests were taken in laboratories other than that of Amir Kabir hospital, were excluded.

Complete red cell count was conducted by cell-count M59, measuring glucose level, using Pars Azma sugar kits and spectrophotometry. Sodium and potassium levels were checked, using flame photometry, and blood urea nitrogen was measured, using Pars Azma kits. Furthermore, urine and stool samples evaluation was carried out manually by a determined individual under specific conditions. Similarity of sampling procedure and laboratory condition was considered.

The test results were recorded, and the range of abnormality was determined based on Nelson textbook of pediatrics. Data were analyzed, using SPSS Version 20. Descriptive statistics comprising of frequency, percentage, mean, and standard deviation were utilized.

Patients' name and information were privately recorded, and no investment was imposed to the families. Tests were taken with the consent of patients and their families. Helsinki declaration was considered in all stages of the research. This research plan was confirmed by the research ethics committee.

\section{Results}

Of the 610 patients with moderate to severe dehydration due to diarrhea and vomiting, 261 (42.78\%) were female, and 472 (77\%) were younger than two years of age. Only two patients had severe dehydration and others had moderate dehydration.

The lowest temperature was 36.5 degrees, and the highest was 40 degrees; 268 patients (43.9\%) had a temperature higher than 37.5 degree, while 342 patients $(56.1 \%)$ had a normal temperature.

Laboratory test measurements are displayed in Table 1.

\section{Discussion}

In this study, most patients were boys younger than two years of age. The average body temperature among patients was 37.6 degrees, and the majority of the patients had normal temperature. In cases with fever, at least one of the following tests: urine, stool or WBC was abnormal.
However, some patients might have been afebrile due to pyretic drug administration by parents at home.

Patients' blood sugar test revealed normal levels of sugar for the majority of the patients. Based on the results, only nine patients were diagnosed with hypoglycemia and eight with hyperglycemia. As the normal percentage of blood sugar is about $97.3 \%$, blood sugar was not measured in these patients. Measuring blood sugar in gastroenteritis patients has not been discussed in pediatrics reference books or other articles.

The majority of the patients had normal serum sodium, which is similar to the study of Yilmaz et al. (8). Hayajneh WA et al. (9) found that serum urea, creatinine, sodium, potassium, and glucose were useful independently in children presenting with gastroenteritis, and serum urea was found to be the best among all. King et al. (7) indicated that serum electrolytes assessment is not necessary for acute diarrhea. However, in our study, only a small proportion of patients had abnormal serum potassium, and hyperkalemia might have been due to a technical error during the sampling (for example, hemolysis due to the child's sudden movement of forearm). Considering the fact that severe gastroenteritis results in severe dehydration, it is logical to evaluate serum electrolytes. In our case, considering the size of our sample, the majority of our patients were suffering from moderate dehydration (1).

According to the results, the majority of the cases had normal WBC, while 44 patients had leukocytosis and 24 were febrile. Therefore, it seems logical to evaluate WBC in febrile gastroenteritis patients. The topic of evaluation of initial tests for moderate to severe diarrhea has been pointed out in previous studies. However, leukocytosis may occur following stress (1). In addition, the study of white blood cells has no validity in proving or disproving diarrhea and vomiting, but it is usually used to determine the underlying causes of fever, like other children's' infectious diseases.

High blood urea nitrogen can be explained and justified with the child's fever and dehydration. It should be noted that most patients with high blood urea nitrogen were febrile (66\%). Teach SJ et al. (10) found that serum $\mathrm{BUN} / \mathrm{cr}$ and serum uric acid were significantly associated with increasing fluid deficit. Yilmaz et al. (8) stated that the amount of blood urea was related to the level of dehydration, while they found no relationship between sodium and degree of dehydration. In the study of Bonadio WA et al. (11), BUN concentration was not a useful assessment for hydration status in children with dehydration due to gastroenteritis.

Almost all the patients had normal hemoglobin, while 131 (21.6\%) had anemia. Complete blood count, especially 
Table 1. Distribution of Laboratory Tests in Patients with Dehydration Due to Gastroenteritis

\begin{tabular}{|c|c|}
\hline Laboratory Test & Frequency (\%) \\
\hline \multicolumn{2}{|l|}{ Blood sugar, 43 - $286 \mathrm{mg} / \mathrm{dL}$} \\
\hline Hypoglycemia & $9(1.5)$ \\
\hline Hyperglycemic & $8(1.2)$ \\
\hline \multicolumn{2}{|l|}{ Sodium, 123 - $171 \mathrm{meq} / \mathrm{dL}$} \\
\hline Hyponatremia & $24(3.9)$ \\
\hline Hypernatremia & $4(0.6)$ \\
\hline \multicolumn{2}{|l|}{ Potassium, 2.6 - $8.0 \mathrm{meq} / \mathrm{dL}$} \\
\hline Hypokalemia & $2(0.3)$ \\
\hline Hyperkalemia & $38(6.1)$ \\
\hline \multicolumn{2}{|l|}{ White blood cell, 2890 - 27000} \\
\hline Leukopenia & $32(5.4)$ \\
\hline Female & $17(53)$ \\
\hline$<2$ years old & $24(75)$ \\
\hline Fever & $18(56)$ \\
\hline Leukocytosis & $44(6.8)$ \\
\hline Female & $16(26)$ \\
\hline$<2$ years old & $33(75)$ \\
\hline Fever & $24(54)$ \\
\hline \multicolumn{2}{|l|}{ Hemoglobin, 6.9 - 21 mg/dL } \\
\hline Anemia & $131(8.3)$ \\
\hline Female & $59(45)$ \\
\hline$<2$ years old & $126(96.2)$ \\
\hline High hemoglobin & $7(1.1)$ \\
\hline \multicolumn{2}{|l|}{ Blood urea nitrogen, maximum = 121} \\
\hline High level & $51(8.3)$ \\
\hline \multicolumn{2}{|l|}{ BUN } \\
\hline Female & $17(33)$ \\
\hline$<2$ years old & $37(64)$ \\
\hline Fever & $34(66)$ \\
\hline \multicolumn{2}{|l|}{ Serum creatinine, maximum = 7.1} \\
\hline High level serum creatinine & $29(4.6)$ \\
\hline \multicolumn{2}{|l|}{ Urine analysis } \\
\hline Abnormal U/A & $57(9.3)$ \\
\hline Female & $39(68)$ \\
\hline$<2$ years old & $49(86)$ \\
\hline Fever & $37(64)$ \\
\hline Positive urine culture & $20(3.2)$ \\
\hline \multicolumn{2}{|l|}{ Stool exams } \\
\hline Abnormal S/E & $102(16.7)$ \\
\hline Female & $40(39)$ \\
\hline$<2$ years old & $72(70)$ \\
\hline Fever & $53(51)$ \\
\hline
\end{tabular}

hemoglobin, is a preliminary evaluation of moderate to severe diarrhea, and is a good criterion for determining the degree of dehydration in the absence of hemorrhagic source $(1,12)$.

Active urine analysis (urinary tract infection) was reported in 57 patients, and 20 had positive urine culture.
Fallahzadeh et al. (13) addressed that urine analysis should be recommended only for children with fever or for girls aged 5 to 15 months. In our study, the majority of the patients with UTI were febrile and were female aged less than two years. In the study of Vargas Origel et al. (14), of the 35 infants, only one (3\%) showed the mentioned relation- 
ship. In addition, urinalysis (U/A) could be used to determine the exact weight of urine in moderate and severe diarrhea as an indicator of hydration status $(1,4,15)$. Caleb K. King et al. (7) found that certain laboratory studies such as complete blood counts, urine, and blood cultures might be important when the underlying diagnosis is unclear or diagnoses other than acute gastroenteritis, such as sepsis or urinary tract infection, are a concern.

Stool cultures are done in cases of dysentery, but not in acute, watery diarrhea for healthy patients (7). Microbiological stool examination is an essential test to detect suspicious conditions for acute and severe diarrhea, and is considered as a significant step in evaluating patients with chronic diarrhea (1). Significant proportion of patients in this study had abnormal stool results, and more than half of them had a fever. It is worth mentioning that almost all of them were male and under two years of age.

Vega et al. (16) suggested that physicians should not rely solely on clinical assessment to rule out severe dehydration in children, and that obtaining a serum bicarbonate may improve the accuracy of predicting serious dehydration.

\subsection{Conclusion}

Serum BUN/cr and serum uric acid were significantly associated with increasing fluid deficit (10). White blood cell test is recommended for children with febrile diarrhea $(1,12)$. Positive stool results on the mentioned factors highlight the importance of conducting the S/E as a diagnostic tool for the diagnosis of the mechanism of diarrhea (inflammatory, or non-inflammatory). According to studies on active U/A and its relationship to diarrhea, it is recommended to perform urinalysis and urine culture for girls under two years of age with fever, who are suffering from diarrhea $(13,14)$.

The results of our study suggest that $\mathrm{Hb}$ should be studied in all patients to treat patients with anemia. As anemia makes a person susceptible to infectious diseases, it is recommended to solve this problem. Additionally, based on the results obtained in our study, there is no need to perform any other routine tests for those patients with diarrhea and moderate dehydration, and decision should be made based on the clinical condition of the patients and their doctors' opinion.

\section{Acknowledgments}

The research team wishes to thank the vice chancellor of research for the financial support and we extend our thanks to children and their parents who contributed to this research.

\section{Footnote}

Funding/Support: The mentioned findings in this essay have been excerpted from MD thesis of Dr. Elahe Ziaei which has been sponsorship by Arak University of Medical Sciences.

\section{References}

1. Bhutta ZA. Acute Gastroenteritis in Children. Philadelphia: Saunder; 2011. pp. 1323-38.

2. Andreh SRC, Martin A. Reading on Diarrhea student manual. Geneva: Switzerland: World health organization; 1992.

3. Friedman JN, Goldman RD, Srivastava R, Parkin PC. Development of a clinical dehydration scale for use in children between 1 and 36 months of age. J Pediatr. 2004;145(2):201-7. doi: 10.1016/j.jpeds.2004.05.035. [PubMed: 15289767].

4. Steiner MJ, Nager AL, Wang VJ. Urine specific gravity and other urinary indices: inaccurate tests for dehydration. Pediatr Emerg Care. 2007;23(5):298-303. doi: 10.1097/01.pec.0000270162.76453.fa. [PubMed: 17505271].

5. United Kingdom. Diarrhoea and Vomiting Caused by Gastroenteritis: Diagnosis, Assessment and Management in Children Younger than 5 Years. London: RCOG Press; 2009.

6. Goldman RD, Friedman JN, Parkin PC. Validation of the clinical dehydration scale for children with acute gastroenteritis. Pediatrics. 2008;122(3):545-9. doi: 10.1542/peds.2007-3141. [PubMed: 18762524].

7. King CK, Glass R, Bresee JS, Duggan C. CDC 2003. Available from: http: //www.cdc.gov/MMWr/preview/mmwrhtml/rr5216a1.htm.

8. Yilmaz K, Karabocuoglu M, Citak A, Uzel N. Evaluation of laboratory tests in dehydrated children with acute gastroenteritis. J Paediatr Child Health. 2002;38(3):226-8. doi: 10.1046/j.1440-1754.2002.00792.x. [PubMed: 12047687].

9. Hayajneh WA, Jdaitawi H, Al Shurman A, Hayajneh YA. Comparison of clinical associations and laboratory abnormalities in children with moderate and severe dehydration. J Pediatr Gastroenterol Nutr. 2010;50(3):290-4. doi: 10.1097/MPG.0b013e31819de85d. [PubMed: 19644395].

10. Teach SJ, Yates EW, Feld LG. Laboratory predictors of fluid deficit in acutely dehydrated children. Clin Pediatr (Phila). 1997;36(7):395-400. doi: 10.1177/000992289703600703. [PubMed: 9241476].

11. Bonadio WA, Hennes HH, Machi J, Madagame E. Efficacy of measuring BUN in assessing children with dehydration due to gastroenteritis. Ann Emerg Med. 1989;18(7):755-7. [PubMed: 2735594].

12. Brunicardi Billiar DH. Shwartz principles of surgery. 17 ed. McGrawHill; 1999.

13. Fallahzadeh $\mathrm{MH}$, Ghane F. Urinary tract infection in infants and children with diarrhoea. Eastern Mediterranean Health J. 2006;12(5):6904.

14. Vargas Origel A, Paz Martinez G, Santos Atherton D. [Urinary infection in children with acute gastroenteritis]. Bol Med Hosp Infant Mex. 1980;37(5):957-62. [PubMed: 7426138].

15. Thakar R, Rath B, Prakash SK, Mittal SK, Talukdar B. Urinary tract infection in infants and young children with diarrhea. Indian Pediatr 2000;37(8):886-9. [PubMed: 10951638].

16. Vega RM, Avner JR. A prospective study of the usefulness of clinical and laboratory parameters for predicting percentage of dehydration in children. Pediatr Emerg Care. 1997;13(3):179-82. [PubMed: 9220501]. 